\title{
Post Publication Review
}

Carlson, B.L., Farrelly, T., Frazer, R., Borthwick, F. Mediating (2015) Tragedy: Facebook, Aboriginal Peoples and Suicide. Australasian Journal of Information Systems, 19. doi:http:// dx.doi.org/ 10.3127/ ajis.v19i0.1174.

\section{Review}

This is a well-considered and informative article detailing the tragedy of premature, Indigenous suicide and the effects of social media. The authors demonstrate a clear need for research in this area, showing for example that Aboriginal suicide is among the highest in the world with children less than 14 years old being nearly eight times more likely to commit suicide than non-Aboriginal Australians. Furthermore, their study identified that Aboriginal people frequently use social media as a communicative tool to mutually support and care for friends and family.

Although the authors revealed that Facebook as a platform broke down barriers for Aboriginal people in accessing formal mental health services, it would have been helpful to readers had they more thoroughly discussed what might be appropriate responses. For instance, one study recently suggested a possible technical solution for rural and remote service providers, where Indigenous populations are frequently higher, because privacy legislation in Australia often hampers the communication of mental health data, hindering appropriate treatment for mental illness (Burmeister, Islam, Dayhew, \& Crichton, 2015). Thus whilst this study's results demonstrate that Facebook offers a further, potentially vital avenue for Aboriginal people to seek help outside formal services, the way in which that can be done compliant with legislation needs further investigation. The use of Facebook appears to be both positive and negative, in that it can be a platform for help-seeking or - giving by overcoming barriers for users, as human connectedness and community is a protective factor for suicide (McKinley, 2016). However, skeptics state that a 'call for help' via Facebook can be an attention-seeking exercise and they lack confidence that Facebook can actually assist. It will be interesting to see if other related initiatives shed further light on this. For instance, the Young and Well Cooperative Research Centre's initiative to build an Internet portal for young people with mental illness, or the online service provided by headspace, or the NSW Kids and Families project 'Health system navigation in the digital age', aimed at improving the way rural kids access health data.

As the authors point out, a promising option in addressing Aboriginal suicide is to engage in already existing effective practices. Facebook's potential to contribute to mental illness, through facilitating racial abuse and encouraging others to suicide, is of great concern. However development and evaluation of culturally appropriate suicide intervention programs, using Facebook as a platform warrants further investigation.

While the methodology has sought to understand the complexities of Indigenous experience, age, gender and other attributes have not been used substantially in interpretation of findings. The authors point out that much of the social media focus is on young people and that their methodology involved 50 interviews with people aged 18-60. However, as they also point out repeatedly, the Aboriginal suicide rates increased from 1970 onwards due to, amongst other causes, systematic oppression. Furthermore, whilst they rightly point to the vulnerability of people exhibiting suicidal ideation, they do not explain why they limited the study to a maximum age of 60 . Suicide rates are high amongst older people in the wider population, and particularly older men. Furthermore, older people are frequently seen as especially vulnerable, because unlike other age groups, they are unlikely to recover from declining functionality, due to the ageing process (Bernoth, Dietsch, Burmeister, \& Schwartz, 2014). Related to this is the fact that the authors highlight the high uptake of social media and mobile technology in Aboriginal communities, but do not differentiate on the basis of age. For instance, psychosocial research in ageing has shown that over the age of 70 people increasingly experience learning difficulties (Baltes \& Smith, 2002), and it has been shown that this is also the case for technology related learning (Burmeister, 2010), which calls into question whether the touted 
benefits the authors claim apply to aged groups they have not considered, namely people older than 60, including older Aboriginal people who experienced the systematic oppression that led to the increasing suicide rates from 1970 onwards.

Further, the observations about social media usage were taken from other studies rather than the specific communities this study was based upon. Whilst it is important to connect this research to that of others working in the area of apps, tablets and Smartphones for suicide prevention (Aguirre, McCoy, \& Roan, 2013), the way the authors have done this makes it difficult to compare their results to those of others.

Given the spread of communities between metropolitan, rural and remote communities, mapping communities to health service providers such as Aboriginal Medical Services, might also have some influence on help-seeking and help-giving behaviours. The authors acknowledge that their participants were more often receiving requests for help and therefore, further work on why they were help-givers might assist in the better understanding of the helpseekers. In suggesting a lack of mental illness such as depression, and that males were more likely to act impulsively in the context of intoxication, the different contributing factors between adults and Aboriginal children under 14, means the understanding about the use of social media is incomplete. Social media is clearly important, as this study demonstrates, but further investigation is required to address its use in reducing the incidence of suicide in Aboriginal communities. Better understanding beliefs about health (Boddington \& Raisanen, 2009), how they link to suicidal ideation, and therefore where appropriate supports might be accessed, would strengthen the important first step this study takes in examining the use of social media in Aboriginal communities.

The authors state that the work reported in the article is in its early stages. Given that many of those most affected by the systematic oppression of past Australian policies are now quite senior in age, not including older people in their sample seems to be an oversight that should be addressed as their study continues. Overall this is an important contribution to theliterature and a topic that needs to be explored in more detail. To successfully combat the alarming rates of suicide in our population more discussion is needed in various people groups: Indigenous, military, law enforcement (McKinley, 2016), and others. The role of digital technologies in that process also needs to be considered.

\section{Amber McKinley}

Charles Sturt University

\section{David Ritchie}

Charles Sturt University

\section{Oliver Burmeister}

Charles Sturt University

oburmeister@csu.edu.au

\section{References}

Aguirre, R. T. P., McCoy, M. K., \& Roan, M. (2013). Development Guidelines from a Study of Suicide Prevention Mobile Applications (Apps). Journal of Technology in Human Services, 31(3), 269-293. doi: 10.1080/ 15228835.2013.814750

Baltes, P. B., \& Smith, J . (2002). New frontiers in thefuture of ageing: From successful ageing of the young old to the dilemmas of the fourth age. Paper presented at the Valencia Forum Valencia, Spain.

Bernoth, M., Dietsch, E., Burmeister, O. K., \& Schwartz, M. (2014). Information Management in Aged Care: Cases of Confidentiality and Elder Abuse. J ournal of Business Ethics, 122, 453-460. doi: 10.1007/ s10551-013-1770-7

Boddington, P. \& Raisanen, U. (2009) Theoretical and Practical Issues in the Definition of Health: Insights from Aboriginal Australia. J . of Medicine and Philosophy, 34(1), 49-67 
Burmeister, O. K. (2010). Websites for seniors: Cognitive accessibility. International J ournal of Emerging Technologies and Society, 8(2), 99-113.

Burmeister, O. K., Islam, M. Z., Dayhew, M., \& Crichton, M. (2015). Enhancing client welfare through better communication of private mental health data between rural service providers. Australasian J ournal of Information Systems, 19, 1-14. doi: http://dx.doi.org/ 10.3127/ ajis.v19i0.1206

McKinley, A. (2016). Solvability and Applied Victimology in New South Wales 1996-2013 (PhD), Bond University, Robina Queensland.

\section{Author Response}

We would like to start by thanking the above authors for their thoughtful and well-informed response to our paper. Their insights are eagerly welcomed - especially considering the subject matter, which is both complex and urgent. The authors identified a number of valid challenges and gaps within our research, which we hope to explore below.

We thought it would be worth opening by explaining in more detail the context in which the paper emerged. The interview data on which the paper is based was collected for a larger research project that explored-broadly-Indigenous Australians' use of social media. This larger project aimed towards developing insight into the diverse practices, meanings and politics of Indigenous peoples' engagement with social media technologies. By talking with approximately a hundred Indigenous people from communities all across Australia-from Sydney to Ceduna, Brisbane to Broome-we hoped to unpack Indigenous social media usage in all its complexity. This qualitative study was primarily interview-based: we thus aimed at obtaining 'thick descriptions' of everyday lived experience rather than broadly representative samples.

Qualitative studies of this nature resist epistemological closure, allowing for the emergence of perhaps unexpected themes. For instance, at the outset of the project, we never imagined that Sorry Business (practices associated with death and loss in many Indigenous cultures) would be such a key issue for Indigenous peoples' experience of social media. Likewise, neither did we predict the extent of Indigenous online activism. We have consequently been able to critically unpack these topics (see Carlson \& Frazer 2015 and Carlson \& Frazer In Press, respectively), which had previously been neglected in the existing literature. Adopting a narrower approach to the terms of the project would likely have precluded these important yet greatly under-explored topics from emerging.

Furthermore, this empirically open approach was, in part, a political decision. Historically, research on Indigenous Australians has often been gravely misdirected-even while generally being well-intentioned. It has been argued that "Australia's Indigenous peoples are the most researched people in the world" (Fredericks 2008; see also Smith 1999). Too often, this research has not been for the benefit for Indigenous peoples themselves. Indeed, research has been directly implicated in increasing both colonial power and the efficacy of Indigenous subjugation. Early anthropological work on Indigenous Australians, for example, often simply confirmed the myth of white superiority. Later, it was also complicit in justifying the forced removal of children from Indigenous communities. In response, and understandably, there exists significant distrust of scientists and other researchers among many Indigenous communities. This is particularly true of approaches that lean towards the 'hard sciences' and thus adopt primarily quantitative methodologies. These approaches have been criticised in some cases for propagating the myth of the distanced, 'neutral' observer, while in fact being influenced heavily by colonial power relations.

For these reasons, we took cue from Torres Strait Islander scholar Martin Nakata (2007), among others (Smith 1999), who calls for the development and adoption of a research paradigm that is both culturally appropriate and relevant for Indigenous Australians-indeed, an adamantly de-colonial research paradigm. For this project, we drew inspiration from Nakata's Indigenous Standpoint Theory, which grants epistemological privilege to Indigenous 
stories, meanings and world-views. In this way, fact and truth are not things that exist in some pure and transcendental form, but are instead continuously (re)produced at the 'cultural interface' of Indigenous people's lived everyday realities.

However, as the above authors have identified, this approach also comes with its own set of limitations. In taking as a point of departure a heterogeneous epistemology, we circumscribe the extent to which any results may be generalisable or comparable across populations. Particularly when it comes to such urgent topics as Indigenous suicide, it does not generate the most rigorous or immediately practicable data.

Instead, we hoped this paper might offer insights into the diverse help-seeking/-giving practices in which Indigenous Australians are already engaged - a topic which has received little academic consideration. We contend that through paying attention to the range of online practices that already exist in Indigenous communities-what we identified as a 'continuum' of practices, ranging from gentle online support to emergency intervention-we may begin to develop approaches to suicide prevention and intervention that are culturally appropriate and relevant. While in our paper we were able to offer initial insights into help-seeking/-giving, we were not able to take the crucial second step of propounding the efficacy of specific practices.

We therefore agree wholeheartedly with the authors that this topic requires much greater attention than the modest insights our paper offered. To help approach this need for greater critical enquiry, the first author of the paper, Associate Professor Bronwyn Carlson, is leading a national study that will explore in detail Indigenous help-seeking and help-giving practices online. Funded by the Australian Research Council for three years commencing this year, the project aims to unlock social media's potential to create vital connections between help-seekers and help-givers. Leveraging on the previous work on Aboriginal and Torres Strait Islander use of social media, the new research project will be the first comprehensive analysis of Aboriginal and Torres Strait Islander help-seeking and help-giving behaviours online.

This larger project will thus aim to address the failings of our initial investigation. As the authors of the response point out, a truly rigorous research project needs to be able to account for differences in age, gender and various other participant attributes. Further, if it wants to effectively reduce the alarming rates of Indigenous suicide, the project's results should offer practical and thoroughly evaluated interventions which can be promoted and implemented by communities, health services, and other interested parties. It is these hopes that we invest in this coming project.

\section{Bronwyn L Carson}

University of Wollongong

\section{Ryan Frazer}

University of Wollongong

rfrazer@uow.edu.au

\section{References}

Carlson, Bronwyn, and Ryan Frazer. "'It's like going to a cemetery and lighting a candle”: Aboriginal Australians, Sorry Business and social media. "AlterNative: An International J ournal of Indigenous Peoples 11.3 (2015).

Carlson, Bronwyn, and Ryan Frazer. Indigenous Activism and Social Media: The Global Response to \#SOSBLAKAUSTRALIA, in Negotiating Digital Citizenship: Control, Contest and Culture, Rowman and Littlefield (In Press).

Fredericks, Bronwyn. "So you want to do oral history with Aboriginal Australians." J ournal of the Oral History Association of Australia 30 (2008): 22-24.

Nakata, Martin N. Disciplining the savages, savaging the disciplines. Aboriginal Studies Press, 2007. 
Smith, Linda Tuhiwai. Decolonizing methodologies: Research and indigenous peoples. Zed books, 1999.

Copyright: (C) 2016 McKinley, Ritchie, Burmeister, Carson \& Frazer. This is an open-access article distributed under the terms of the Creative Commons Attribution-NonCommercial 3.0 Australia License, which permits non-commercial use, distribution, and reproduction in any medium, provided the original author and AJ IS are credited.

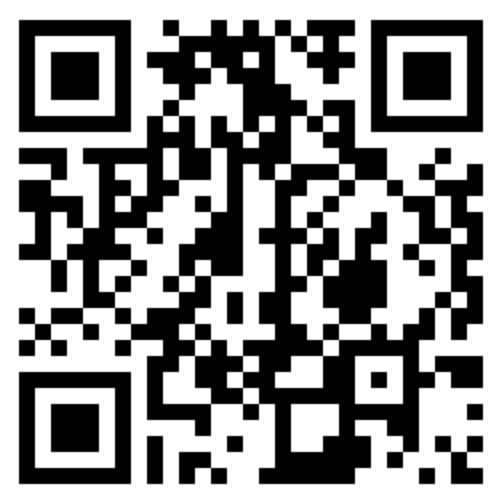

